Devel opment of $\mathrm{Hi}$ gh- Fr equency, $\mathrm{Hi}$ ghl y St abl e Gyr ot rons as MII i met er to Submil i meter have Radi at i on Sources

\begin{tabular}{|l|l|}
\hline 著者 & $\begin{array}{l}\text { I DEHARA Toshi taka, M TSUDO Sei t ar o, OGAWA } \\
\text { I sami }\end{array}$ \\
\hline $\begin{array}{l}\text { j our nal or } \\
\text { publ i cat i on ti t l e }\end{array}$ & I EEE Tr ansact i ons on PI asma Sci ence \\
\hline vol une & 32 \\
\hline number & 3 \\
\hline page r ange & $910-916$ \\
\hline year & 2004 06 \\
\hline URL & ht t p: //hdl . handl e. net /10098/1602 \\
\hline
\end{tabular}




\title{
Development of High-Frequency, Highly Stable Gyrotrons as Millimeter to Submillimeter Wave Radiation Sources
}

\author{
Toshitaka Idehara, Seitaro Mitsudo, and Isamu Ogawa
}

\begin{abstract}
High-frequency, frequency-tunable, medium-power gyrotrons (Gyrotron FU series) are being developed in Fukui University as millimeter to submillimeter wave sources. The gyrotron series has achieved frequency tuneability in a wide range from 38 to $889 \mathrm{GHz}$ and medium output power from $0.1 \mathrm{~kW}$ to several kilowatts in pulse mode (the pulse width is $1 \mathrm{~ms}$ and repetition rate 1 $\mathrm{Hz}$ ) and several tens of watts in $\mathrm{CW}$ mode. Stabilizations of both amplitude and frequency have been achieved, for the application to many fields. High purity mode operations by installation of carefully designed cavity were also achieved for highly efficient conversion to Gaussian beam. In this paper, the advances in recent few years are summarized.
\end{abstract}

Index Terms-Gyrotron, millimeter and submillimeter waves.

\section{INTRODUCTION}

$\mathbf{S}$ UBMILLIMETER wave gyrotrons in the Gyrotron FU series at the Research Center for Development of Far-Infrared Region, Fukui University (FIR FU) have been developed [1] as short millimeter to submillimeter wave radiation sources and applied successfully to plasma scattering measurements [2], ESR experiments [3], and a new medical technology [4]. The gyrotron FU series contains frequency step-tunable sources from 38 to $889 \mathrm{GHz}$ and has many advantages, for example, continuous wave (CW) operation on long time scales [5], modulations of amplitude [6], and frequency [7] and fast switching of frequency. [8] These features are useful and convenient for application of the submillimeter wave gyrotron series to many fields.

The advances in recent few years are summarized as follows.

1) Stabilization of the frequency by the phase lock control of beam energy

The output frequency of Gyrotron FU IV is stabilized by controlling high-voltage power supplies for both the gun anode and cathode. In addition, we apply the phase lock control to the beam energy to achieve higher stabilization of the frequency. Both the half width of the frequency spectrum $\triangle f$ and the frequency fluctuation $\delta f$ are decreased to less than $0.3 \mathrm{kHz}$ and $0.1 \mathrm{kHz}$, respec-

\footnotetext{
Manuscript received August 17, 2003; revised November 19, 2003. This work was supported in part by the Ministry of Education, Science, Sports and Culture in Japan under the Grant in Aid for International Research Program, and in part by the Deutsche Forschungsgemeinschaft through the German Japanese Scientific Research Program.

T. Idehara and S. Mitsudo are with the Research Center for Development of Far-Infrared Region, Fukui University, Fukui 910-8507, Japan, (Idehara@ @ir.fukui-u.ac.jp).

I. Ogawa is with the Cryogenic Laboratory, Faculty of Engineering, Fukui University, Fukui 910-8507, Japan.

Digital Object Identifier 10.1109/TPS.2004.827599
}

tively. This means that both $\triangle f / f$ and $\delta f / f$ are lower than $1 \cdot 10^{-9}$ and $3 \cdot 10^{-10}$.

2) Accurate frequency measurement of a submillimeter wave gyrotron output using a FIR laser as a reference [9]

Accurate measurement of gyrotron frequency has been carried out by using a far-infrared (FIR) molecular laser as a reference. Highly stable operation of a FIR laser enables us to make the resolution of frequency measurement so precisely that both the frequency width $\triangle f$ and the frequency fluctuation level $\delta f$ can be studied within the accuracy of $1 \mathrm{kHz}$ or less. When our submillimeter wave gyrotron operates in a free-running $\mathrm{CW}$ mode, the observed frequency width $\triangle f$ is around $2 \mathrm{kHz}$ and the fluctuation level $\delta f 10 \mathrm{kHz}$. The study was carried out under nice collaboration with Prof. S. Okajima from Chubu University.

3) High purity mode operation by installation of a carefully designed cavity

A cavity is designed for high-purity mode operations under collaboration with University of Stuttgart and FZK in Germany and installed in Gyrotron FU VA. [10] The gyrotron emits many modes with high purities. For almost all modes, the purity is higher than $90 \%$.

In this paper, such recent advances are reviewed in detail.

\section{FIRST EXPERIMENT ON THE HIGH-FREQUENCY \\ STABILIZATION OF A SUBMILLIMETER WAVE GYROTRON BY \\ Phase Lock CONTROL OF ITS ElECTRON BEAM ENERGY}

We have already achieved frequency modulation using Gyrotron FU IV [7]. In this case, the electron beam energy is first changed by the modulation of the cathode or body potential. This results in the modulation of the cyclotron frequency following the formula $\omega_{\mathrm{c}}=e B / m_{0} \gamma$, where $e$ and $m_{0}$ are the electric charge and the rest mass of an electron, gamma is the relativistic factor and $B$ is the applied magnetic field.

Gyrotrons operate at the electron cyclotron frequency and its harmonics. Finally, the output frequency of gyrotron can be modulated. Frequency modulation recently resulted in success under the following conditions; the gyrotron frequency is $301.98 \mathrm{GHz}$, the operation cavity mode is $\mathrm{TE}_{031}$, the maximum amplitude of the modulation is $30 \mathrm{MHz}$. The modulation frequency can be varied up to $40 \mathrm{kHz}$. This may be useful to apply for communications in the submillimeter wavelength region and for scientific applications.

The success of the frequency modulation suggests the possibility of a high stabilization of the output frequency through 


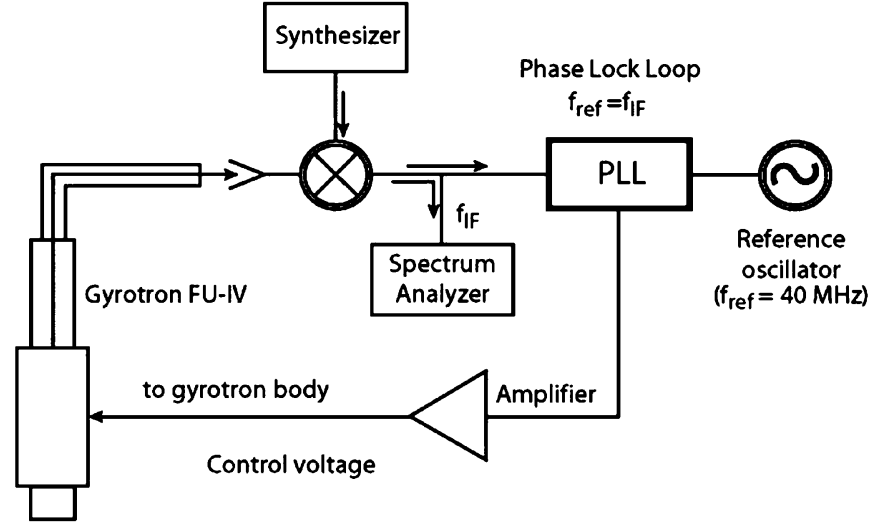

Fig. 1. Block diagram of phase lock stabilization system for a gyrotron frequency. The modulation sensitivity is $16 \mathrm{kHz} / \mathrm{volt}$.

the phase lock control of the electron beam energy. We tried an experiment on this subject. Fig. 1 shows a block diagram of the experimental setup. The Gyrotron FU IV operates in CW mode [5] using stabilized high voltage power supplies for both the gun anode and cathode. The output frequency $f_{0}$ and power $P_{0}$ are $301.128 \mathrm{GHz}$ and around $20 \mathrm{~W}$, respectively. The stabilizations of frequency $\delta f / f_{0}$ and output power $\delta P / P_{0}$ are around $10^{-7}$ and $10^{-4}$. The output power from the Gyrotron FU IV is transmitted through the waveguide system and fed by a harmonic mixer. In the mixer, the gyrotron power is mixed with the signal from a synthesizer. The $40 \mathrm{MHz}$ IF signal is fed on the phase lock loop circuit (PLL) and compared with the signal from a reference (crystal) oscillator with a frequency of $40 \mathrm{MHz}$. The output voltage from the PLL is amplified and then applied to the gyrotron body.

The body includes a gyrotron cavity. Therefore, the energy of the electron beam injected into the cavity is modulated by the feedback control voltage, which is generated in the PLL. The modulation sensitivity is adjusted at $1.6 \cdot 10^{4} \mathrm{~Hz} / \mathrm{V}$, by controlling the amplification factor. This value is much smaller than those of other conventional oscillators, for example, Gunn diodes, klystrons, backward oscillators, etc., because gyrotrons are essentially frequency constant oscillators.

Fig. 2 shows a typically observed frequency spectra with phase lock control (upper figure) and without phase lock control (lower figure). During the phase lock control, the half value width of the frequency spectrum $\triangle f$ and frequency fluctuation width $\delta f$ are both smaller than $0.3 \mathrm{kHz}$ and $0.1 \mathrm{kHz}$, respectively. This means that $\triangle f / f$ is smaller than $1 \cdot 10^{-9}$ and $\delta f / \mathrm{f} 3 \cdot 10^{-10}$. On the other hand, without phase lock control, the half value width $\triangle f$ is around $5 \mathrm{kHz}$. The phase lock control of the electron beam energy gives the gyrotron operation its stability. This is the first experimental result on the phase lock stabilization of the frequency in a gyrotron.

Fig. 3(b) shows the modulation voltage $\triangle V_{\mathrm{b}}$ applied on the gyrotron body as a function of time, while Fig. 3(a) is the corresponding observed frequency spectrum. It can be seen that the output frequency is being locked by the modulation of the body potential. The amplitude of the modulation is only a few volts.

Such high stability and low fluctuation level are both enough for application of the gyrotron to plasma scattering measure-
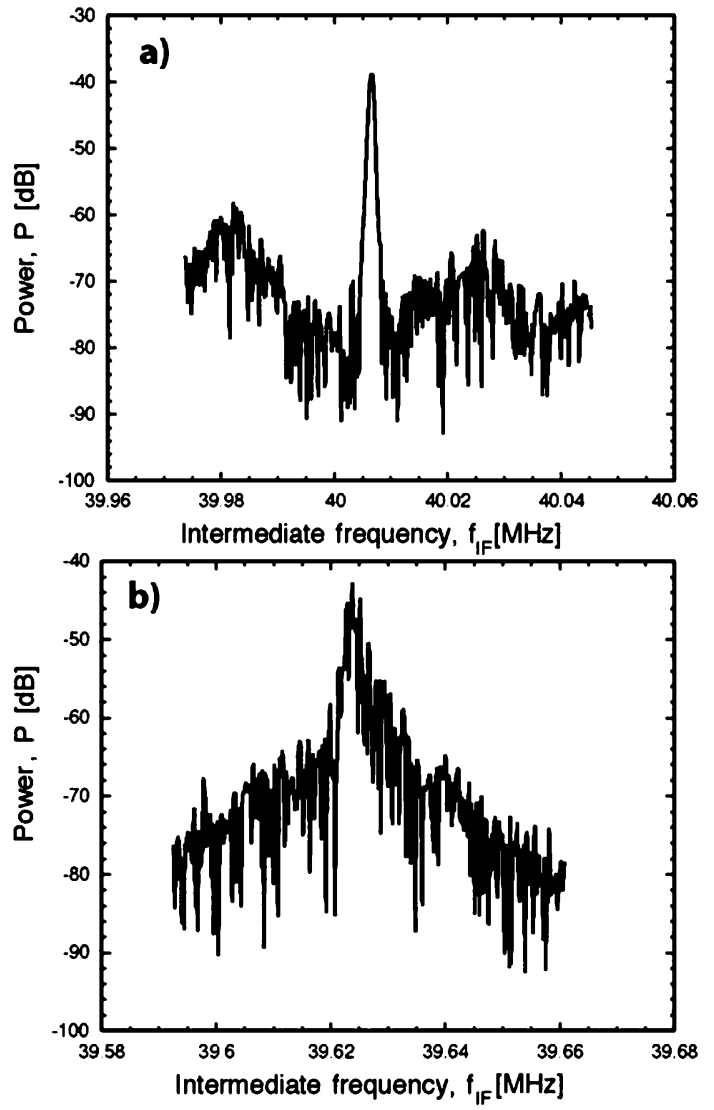

Fig. 2. Observed frequency spectra of Gyrotron FU IV output (a) with phase lock control and (b) without phase lock control. Output frequency of the gyrotron $f_{\mathrm{g}}=301.128 \mathrm{GHz}$. Full sweep time $600 \mathrm{~ms}$. $5 \mathrm{kHz} / \mathrm{div}$.

ment and even ERS spectroscopy. Of course, similar frequency stabilizations have been achieved for other conventional low power radiation sources listed above. These sources are being used for spectroscopies in various scientific research fields. The stability achieved by the gyrotron is compared with or higher than those of other conventional radiation sources.

In addition, the gyrotrons in Gyrotron FU series in FIR FU are also frequency step-tunable, relatively high power radiation sources. The frequency can be changed in the wide range from $38 \mathrm{GHz}$ to $889 \mathrm{GHz}$. The output power is several tens of watts, when operated in $\mathrm{CW}$ mode. Therefore, a high power, highly stable submillimeter wave source was just realized.

\section{AcCuRATe Frequency Measurement of a SUBMILLIMETER WAVE GYROTRON OUTPUT USING A FIR LASER AS A REFERENCE}

The results of accurate measurement of gyrotron frequency using a FIR laser as a reference have already been published [9]. Fig. 4 shows the experimental apparatus. Gyrotron FU IV operates in CW mode. The operation frequency is around 295 $\mathrm{GHz}$. The output power is around $20 \mathrm{~W}$. It is transmitted through an oversized circular waveguide and fed on a Shottky barrier diode. The real power fed on the diode is $70 \mathrm{~mW}$. The diode operates as a harmonic mixer.

After mixing with the signal from a FIR laser, an intermediate (IF) signal is observed on a spectrum analyzer. The IF signal includes a frequency component of four times of gyrotron fre- 


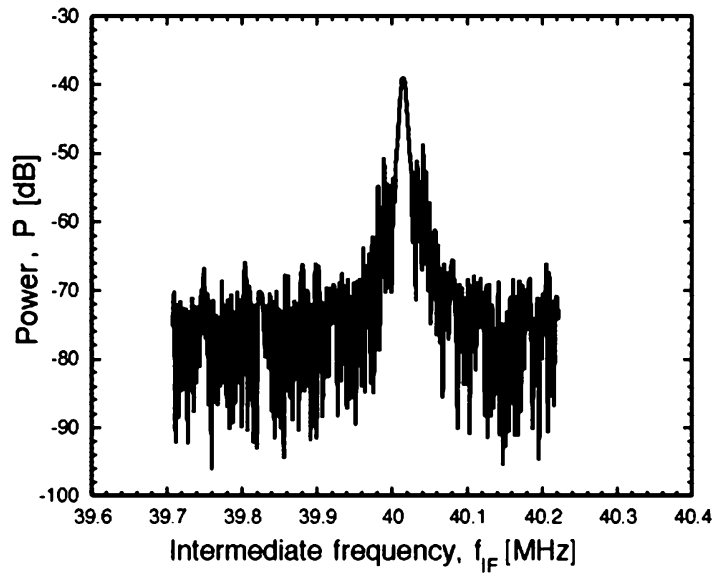

(a)

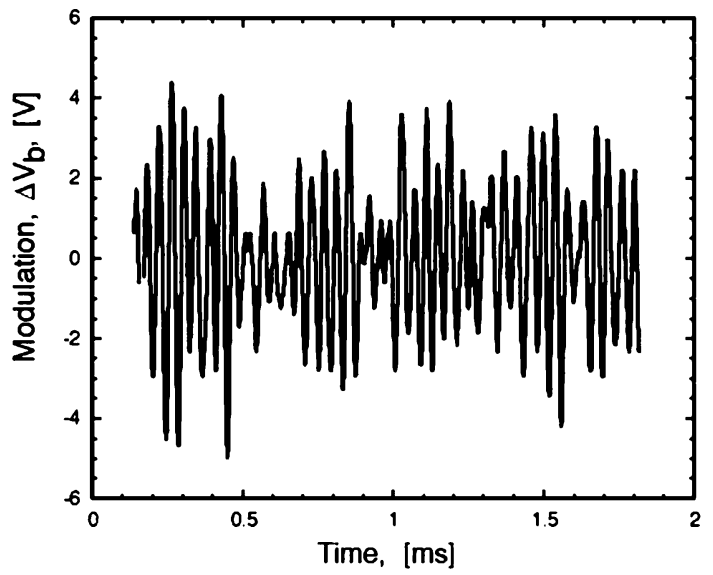

(b)

Fig. 3. (a) Frequency spectrum during phase lock control of the body potential. Output frequency of the gyrotron $f_{\mathrm{g}}=301.128 \mathrm{GHz}$. Full sweep time $10 \mathrm{~ms}$. $20 \mathrm{kHz} /$ div. (b) Corresponding modulation voltage $\Delta V_{\mathrm{b}}$ fed on the body as a function of time.

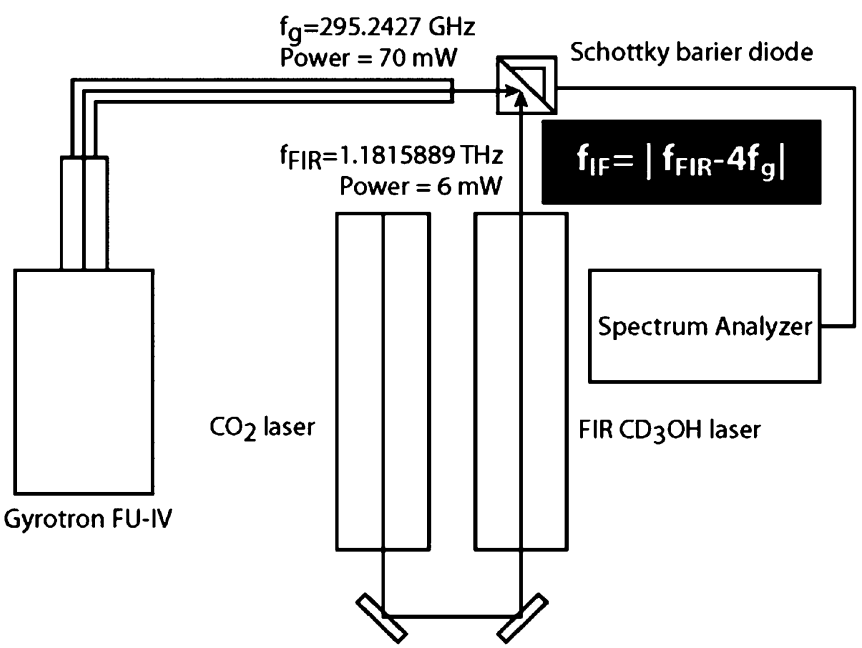

Fig. 4. Block diagram of frequency measurement system using a FIR laser as a reference.

quency $4 \mathrm{f}_{\mathrm{g}}$. Here, we use $\mathrm{CD}_{3} \mathrm{OH}$ laser excited by a $\mathrm{CO}_{2}$ laser. The frequency $f_{\text {FIR }}$ is $1.1815889 \mathrm{THz}$. And the incident power fed onto the diode is $6 \mathrm{~mW}$.

Before measurement of gyrotron frequency using a FIR laser, we tried it by the use of a synthesizer as a reference. Fig. 5 shows

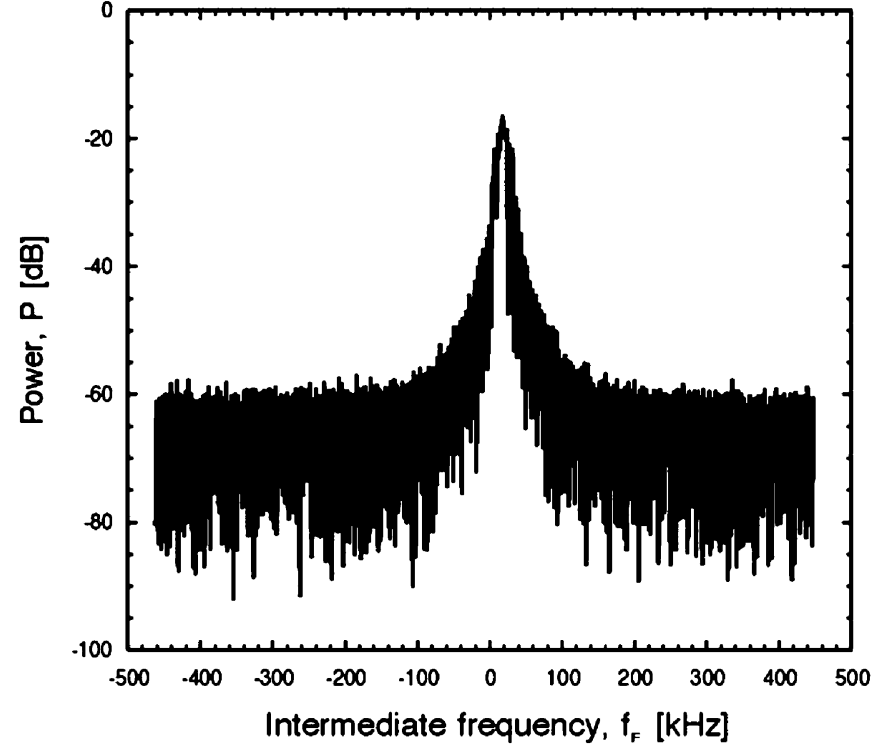

Fig. 5. Frequency spectrum of Gyrotron FU IV output measured by using a synthesizer as a reference. Output frequency of the gyrotron $f_{\mathrm{g}}=295.2427$ $\mathrm{GHz}$.

a result of the measurement. This frequency spectrum is one of the IF signal, which is obtained by the mixing of gyrotron output with 17 th harmonic of the synthesizer output whose frequency is $f_{\text {shy }}$. Therefore, the signal with the frequency $f=\left|f_{\mathrm{g}}-17 f_{\text {shy }}\right|$ is analyzed. On the frequency spectrum, the half value width $\triangle f$ can be observed. It is seen that $\triangle f$ around $20 \mathrm{kHz}$ or wider. This frequency width of IF signal comes mainly from the frequency width of the synthesizer. In this measurement, both the feature of frequency width and frequency fluctuation of the gyrotron can not be resolved so accurately.

Fig. 6 shows frequency spectra of IF signal, which are observed by the use of a FIR laser as a reference [9]. In Fig. 6(a), the total sweep time is $10 \mathrm{~ms}$. In this case, the observed frequency width is smaller than $10 \mathrm{kHz}$. A real frequency width of gyrotron output $\triangle f$ is one fourth of it. Therefore, $\triangle f$ is around $2 \mathrm{kHz}$.

In Fig. 6(b), the total sweep time is $10 \mathrm{~s}$. In the figure, some frequency fluctuations are observed. The width of frequency fluctuation is around $40 \mathrm{kHz}$. So, the real frequency fluctuation of the gyrotron output is around $10 \mathrm{kHz}$. Such a frequency fluctuation comes mainly from the fluctuation of high voltage power supplies for an anode and a cathode of electron gun. We should stabilize the power supplies for removing the frequency fluctuation. Highly stable operation of a FIR laser enables us to make such an accurate measurement of gyrotron frequency. The frequency resolution of the measurement is much higher than 1 $\mathrm{kHz}$. This is a convenient and useful tool for development of high-quality gyrotrons with stable frequency.

\section{High Purity Mode Operation by InStallation of Carefully Designed CaVity}

Gyrotron FU VA consists of an $8 \mathrm{~T}$ liquid-helium free, superconducting magnet and a demountable tube. In the tube, every component, such as the triode magnetron injection gun, the resonant cavity, the transmission waveguide and the vacuum 

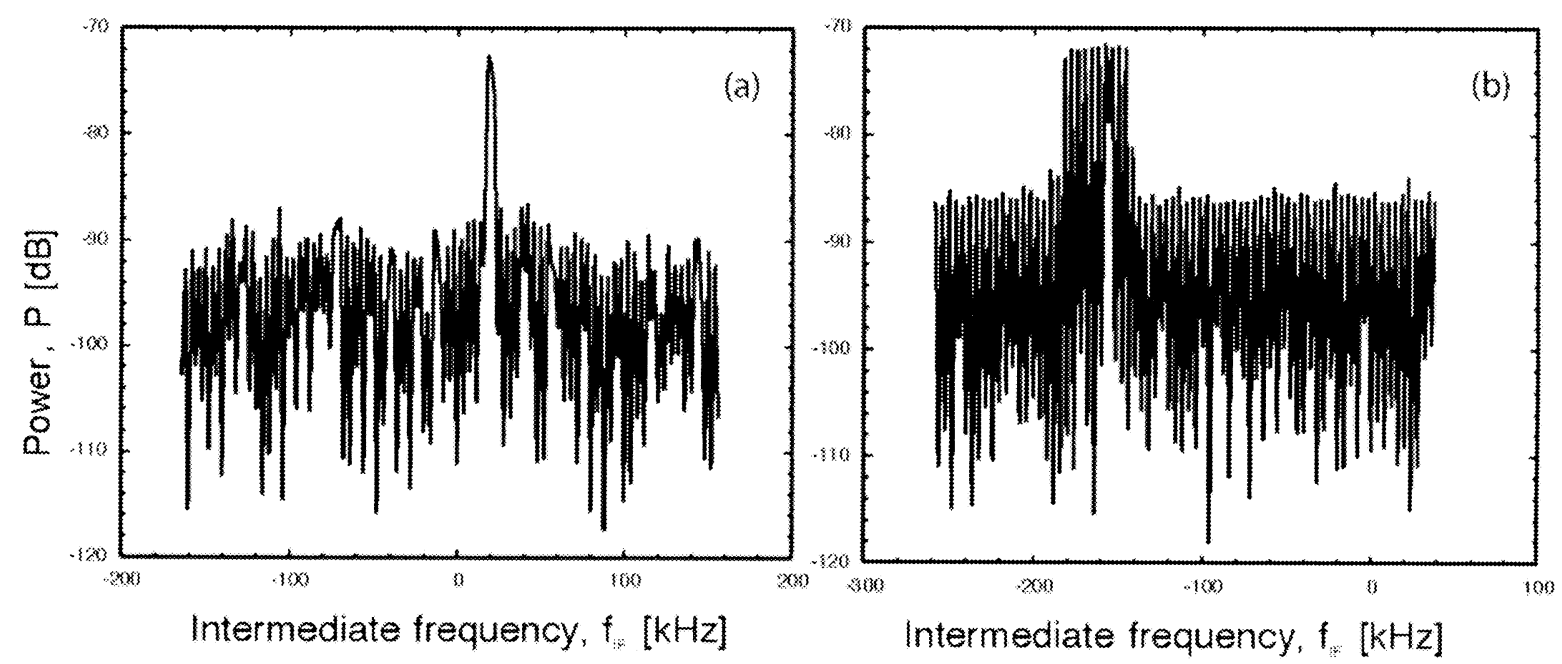

Fig. 6. Frequency spectrum of Gyrotron FU IV output measured by using a FIR laser as a reference. (a) Full sweep time $10 \mathrm{~ms}$ and (b) $10 \mathrm{~s}$.

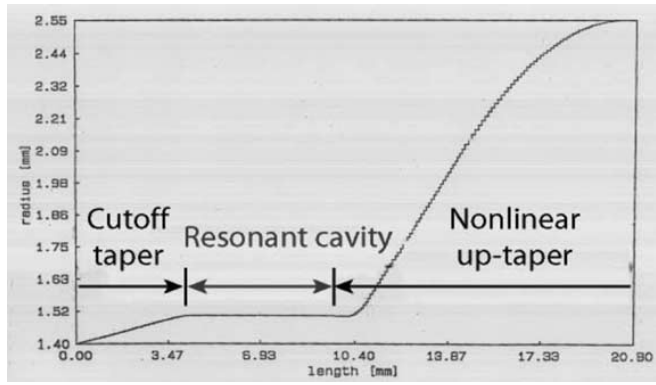

Fig. 7. Profile of the resonant cavity installed in Gyrotron FU VA.

window can be replaced easily. The work described here was already publicshed elsewhere. [10] A cavity designed under collaboration with Karlsruhe Research Center and Stuttgart University to suppress mode conversion into parasitic modes and to get high-purity mode operation was installed. The profile of the cavity is shown in Fig. 7. At the output end of the cavity, there was a small iris for increasing the quality factor in order to allow low starting currents and a nonlinear up-taper for the suppression of any conversion to parasitic modes. The high-frequency electromagnetic wave then travels along a waveguide of diameter $18 \mathrm{~mm}$ toward the vacuum window. The whole tube is pumped out first by a turbo-molecular pump and then by an ion pump.

The patterns of the output power radiated from the window are measured by a pyroelectric detector array moving in a horizontal plane ( $x-y$ plane) at some distance above the gyrotron window. A fixed pyroelectric detector set outside the scanned area provided a signal to normalize the signal from the array. These signals are fed into a computer which converts the data into a 3-dimensional plot of the radiation patterns and a lot of equi-power contours in the $x-y$ plane.

In Fig. 8, radiation patterns obtained by the array of pyroelectric detectors are shown for several cavity modes, $\mathrm{TE}_{221}(128 \mathrm{GHz}), \mathrm{TE}_{021}(132 \mathrm{GHz}), \mathrm{TE}_{321}(152 \mathrm{GHz})$, $\mathrm{TE}_{131}(162 \mathrm{GHz}), \mathrm{TE}_{421}(175 \mathrm{GHz}), \mathrm{TE}_{231}(190 \mathrm{GHz})$, $\mathrm{TE}_{031}(194.6 \mathrm{GHz}), \mathrm{TE}_{521}(199 \mathrm{GHz})$, and $\mathrm{TE}_{331}(215 \mathrm{GHz})$. Each pattern looks pure, in a single mode.
The emission patterns for $\mathrm{TE}_{221}, \mathrm{TE}_{321}, \mathrm{TE}_{131}, \mathrm{TE}_{421}$, $\mathrm{TE}_{231}, \mathrm{TE}_{521}$, and $\mathrm{TE}_{331}$ modes appear as standing waves in the azimuthal direction. This has been observed previously. [3], [4] The actual ratio of the two oppositely rotating modes can be determined by a voltage standing wave ratio (VSWR) $\rho$ measurement following the azimuthal direction $\theta$. As well-known, $\rho$. is defined as

$$
\rho .=\frac{P_{\max }}{P_{\min }}=\frac{\left(A_{1}+A_{2}\right)^{2}}{\left(A_{1}-A_{2}\right)^{2}}
$$

where $P_{\max }$ and $P_{\min }$ are maximum and minimum power measured following $\theta$ direction for the outside peaks and $A_{1}$ and $A_{2}$ are amplitudes of rotating and counter-rotating modes.

Table I shows VSWRs estimated from the observed emission patterns and the ratios of both modes determined from VSWRs. We are studying the reason why the ratio is so close to one that the mode appears stationary in the azimuthal direction.

Calculations of the resonant frequency and quality factor have been carried out using a scattering matrix formalism. These calculations took into account the complete gyrotron geometry including the slots on the output waveguide for pumping, the connection between the cavity and the collector and the vacuum window. [5] Table II summarizes the results for several main cavity modes $\mathrm{TE}_{0 \mathrm{~m} 1}$ and $\mathrm{TE}_{1 \mathrm{~m} 1}$. As can be seen, in almost all cases, more than $95 \%$ of the power reaching the window remains in the main cavity mode.

Fig. 9 shows the calculated results of emission patterns for $\mathrm{TE}_{221}, \mathrm{TE}_{021}, \mathrm{TE}_{321}, \mathrm{TE}_{131}, \mathrm{TE}_{421}, \mathrm{TE}_{231}$, and $\mathrm{TE}_{031}$ cavity modes including the parasitic modes, in the small amounts predicted by the scattering matrix calculations. In the cases of $\mathrm{TE}_{221}$, $\mathrm{TE}_{321}, \mathrm{TE}_{131}, \mathrm{TE}_{421}$, and $\mathrm{TE}_{231}$ modes, the counter-rotating modes are also included with the same ratios as determined from the observed patterns. These calculated patterns are in good agreement with the observed patterns. High purity mode outputs like these are desirable for conversion to Gaussian-like beams. In particular, $\mathrm{TE}_{0 \mathrm{n}}$ and $\mathrm{TE}_{1 \mathrm{n}}$ waveguide modes can be converted easily to Gaussian-like beams by a quasioptical system including a Vlasov antenna and focusing mirrors. [6] 


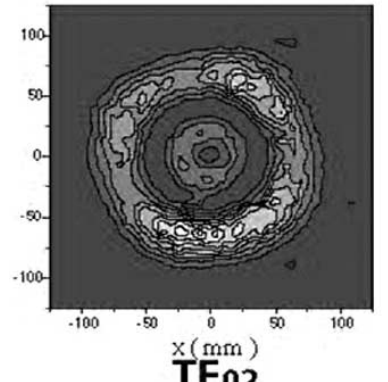

TE02

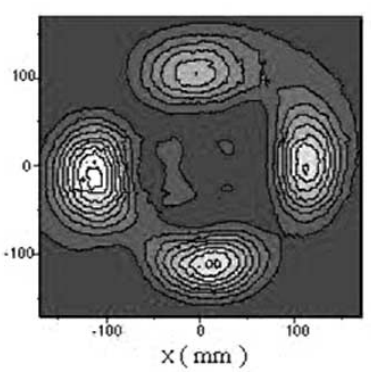

TE22

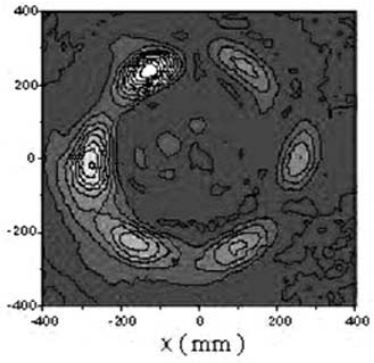

TE33

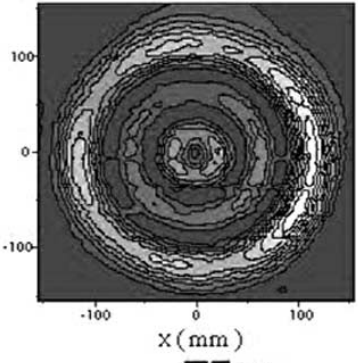

TE03

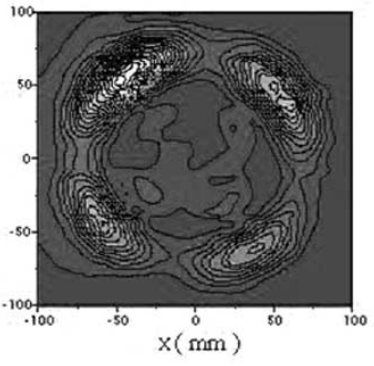

TE23

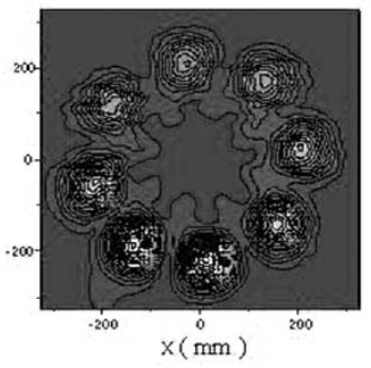

T042

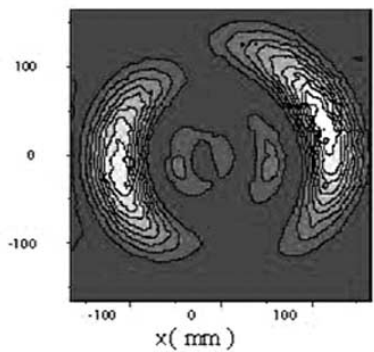

TE13

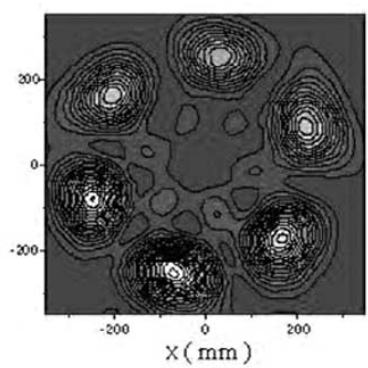

TE32

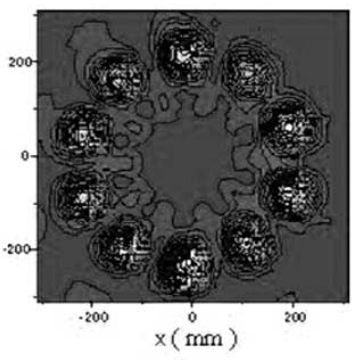

TE52

Fig. 8. Emission patterns for several cavity modes, obtained by an array of pyroelectric detectors moving in the $x-y$ plane located at some distance above the output window.

TABLE I

VSWR IN THE AZIMUTHAL DiRECTION, WHICH Is ESTIMATED FROM THE OBSERVED EMISSION PATTERNS, AND THE RATIO OF THE TWO OPPOSITELY ROTATING MODES DETERMINED FROM THE VSWR

\begin{tabular}{|c|c|c|c|}
\hline $\begin{array}{l}\text { Main cavity } \\
\text { mode }\end{array}$ & $\begin{array}{c}\text { VSWR } \\
\square\end{array}$ & $\begin{array}{l}\text { Ratio of rotating } \\
\text { mode } \\
\text { (in power) } A_{1} \\
\end{array}$ & $\begin{array}{l}\text { Ratio of counter- } \\
\text { rotating mode } \\
\text { (in power) } \boldsymbol{A}_{2} \\
\end{array}$ \\
\hline $\mathbf{T E}_{221}$ & 6.25 & 70 percent & 30 percent \\
\hline $\mathbf{T E}_{321}$ & 20.66 & 61 & 39 \\
\hline $\mathbf{T E}_{131}$ & 5.17 & 72 & 28 \\
\hline $\mathbf{T E}_{421}$ & 7.72 & 68 & 32 \\
\hline $\mathbf{T E}_{231}$ & 4.73 & 73 & 27 \\
\hline$T E_{521}$ & 12.76 & 64 & 36 \\
\hline $\mathbf{T E}_{331}$ & 2.30 & 83 & 17 \\
\hline
\end{tabular}

\section{CONCLUSION}

For many applications of submillimeter wave gyrotron FU series, output frequency of Gyrotron FU IV was highly stabilized by a phase lock control of electron beam energy. The half value width of the frequency spectrum $\triangle f / f$ and the fluctuation level of the frequency $\delta f / f$ are smaller than $1 \cdot 10^{-9}$ and $3 \cdot 10^{-10}$, respectively. First experiment on the phase lock control of elec-
TABLE II

Frequencies $f$, Quality Factors $Q$, AND Mode PuRity Factors $\eta$ From THE SCATtering Matrix CAlCUlations

\begin{tabular}{c|c|c|c}
\hline $\begin{array}{c}\text { Cavity } \\
\text { mode }\end{array}$ & Frequency $f(\mathrm{GHz})$ & Quality factor Q & $\begin{array}{c}\text { Mode purity } \\
(\%)\end{array}$ \\
\hline $\mathrm{TE}_{01}$ & 73.85 & 251 & 97.99 \\
\hline $\mathrm{TE}_{12}$ & 102.36 & 1020 & 98.54 \\
\hline $\mathrm{TE}_{02}$ & 134.40 & 2910 & 97.26 \\
\hline $\mathrm{TE}_{13}$ & 163.37 & 3109 & 99.42 \\
\hline $\mathrm{TE}_{03}$ & 194.57 & 2603 & 98.02 \\
\hline $\mathrm{TE}_{04}$ & 254.63 & 11569 & 98.49 \\
\hline $\mathrm{TE}_{16}$ & 344.13 & 27712 & 87.65 \\
\hline $\mathrm{TE}_{06}$ & 374.63 & 101064 & 93.85 \\
\hline
\end{tabular}

tron beam energy for high stabilization of the gyrotron has been achieved.

By the use of a FIR laser as a reference, we have succeeded the highly accurate measurement of gyrotron frequency. The frequency resolution of the measurement is higher than $1 \mathrm{kHz}$. During free-running $\mathrm{CW}$ operation, real frequency width $\triangle f$ and the frequency fluctuation of the gyrotron $\delta f$ are around 2 and $10 \mathrm{kHz}$, respectively. Such an accurate measurement of fre- 


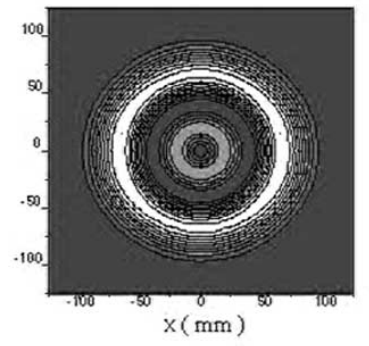

TE02

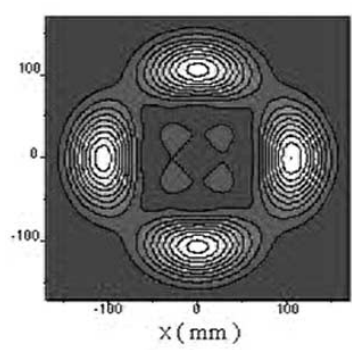

TE22

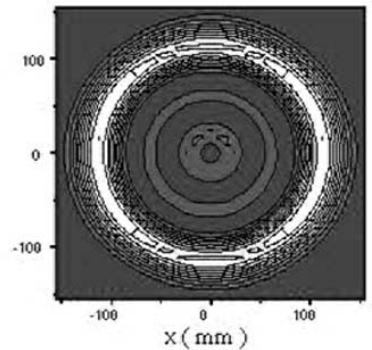

TE03

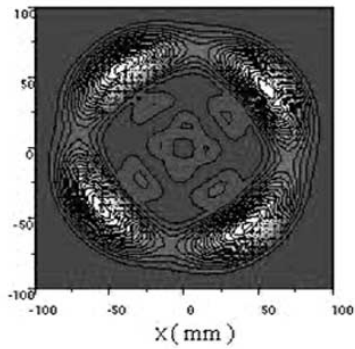

TE23

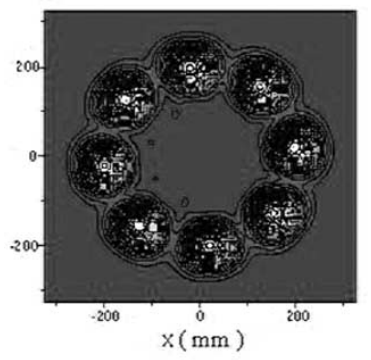

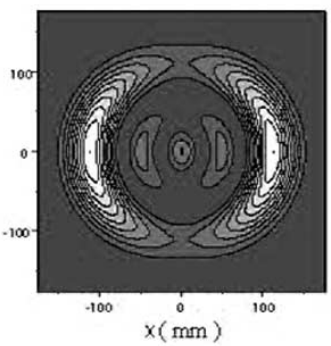

TE13

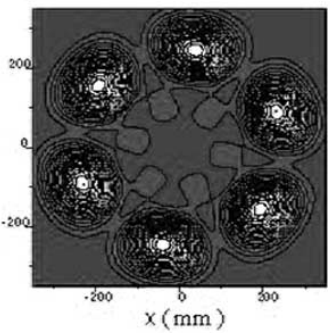

TE32

TE42

Fig. 9. Calculated emission patterns for several cavity modes, including parasitic modes and counter-rotating modes (see text).

quency will be a powerful tool for supporting our future development of high-quality gyrotron with stable frequency, which is useful for spectroscopy in many fields.

Gyrotron FU VA with its carefully designed cavity has achieved high-purity mode operation in several cavity modes. In the case of the $\mathrm{TE}_{221}, \mathrm{TE}_{021}, \mathrm{TE}_{321}, \mathrm{TE}_{131}, \mathrm{TE}_{421}$, $\mathrm{TE}_{231}$, and $\mathrm{TE}_{031}$ cavity modes, the calculations based on a scattering matrix formalism are in good agreement with the observed patterns. The next step, now underway, is to employ a quasioptical system for conversion from the high-purity mode to a Gaussian-like beam.

\section{ACKNOWLEDGMENT}

The author would like to thank Prof. S. Okajima from Chubu University, Kasugai Aichi, Japan, Prof. M. Thumm from Karlsruhe Research Center, Karlsruhe, Germany, Dr. D. Wagner from Stuttgart University, Stuttgart, Germany, and Dr. G. F. Brand from University of Sydney, Sydney, Australia, for their close cooperations on development of high-frequency gyrotrn FU series.

\section{REFERENCES}

[1] T. Idehara, I. Ogawa, S. Mitsudo, M. Pereyaslavets, N. Nishida, and K. Yoshida, "Development of frequency tunable, medium power gyrotrons (Gyrotron FU series) as submillimeter wave radiation sources," IEEE Trans. Plasma Sci., vol. 27, p. 340, 1999.

[2] I. Ogawa, K. Yoshisue, H. Ibe, T. Idehara, and K. Kawahata, "Long pulse operation of a submillimeter wave gyrotron and its application to plasma scattering measurement," Rev. Sci. Instrum., vol. 65, p. 1788, 1994.

[3] S. Mitsudo, Aripin, T. Shirai, T. Matsuda, T. Kanemaki, and T. Idehara, "High power, frequency tunable, submillimeter wave ESR device using a gyrotron as a radiation source," Int. J. Infrared Millimeter Waves, vol. 21, p. 661,2000

[4] T. Tatsukawa, A. Doi, M. Teranaka, H. Takashima, F. Goda, T. Idehara, I. Ogawa, S. Mitsudo, and T. Kanemaki, "Development of submillimeter wave catheter transmitting a gyrotron output for irradiation on living bodies," Int. J. Infrared Millimeter Waves, vol. 21, p. 1155, 2000.

[5] T. Idehara, K. Yoshida, N. Nishida, I. Ogawa, M. Pereyaslavets, and T. Tatsukawa, "CW operation of a submillimeter wave gyrotron (Gyrotron FU IV) for high stability of the output frequency," Int. J. Infrared Millimeter Waves, vol. 19, p. 793, 1998.

[6] I. Idehara, Y. Shimizu, S. Makino, K. Ichikawa, T. Tatsukawa, I. Ogawa, and G. F. Brand, "Amplitude modulation of submillimeter wave gyrotrons," Int. J. Infrared Millimeter Waves, vol. 18, p. 391, 1997.

[7] I. Idehara, M. Pereyaslavets, N. Nishida, K. Yoshida, and I. Ogawa, "Frequency modulation in a submillimeter wave gyrotron," Phys. Rev. Lett., vol. 81, p. 1973, 1998. 
[8] T. Idehara, Y. Shimizu, I. Ogawa, T. Tatsukawa, and G. F. Brand, "Rapid frequency step-switching in submillimeter wave gyrotrons (Gyrotron FU II and FU IV)," Phys. Plasmas, vol. 6, p. 2612, 1999.

[9] T. Idehara, S. Mitsudo, S. Saito, I. Ogawa, and S. Okajima, "Accurate frequency measurement of a submillimeter wave gyrotron output using a far-infrared laser as a reference," Rev. Sci. Instrum., vol. 74, p. 2860, 2003.

[10] T. Idehara, I. Ogawa, S. Maeda, R. Pavlichenko, S. Mitsudo, D. Wagner, and M. Thumm, "Observation of mode patterns for high purity mode operation in the submillimeter wave gyrotron FU VA," Int. J. Infrared Millimeter Waves, vol. 23, p. 1287, 2002.

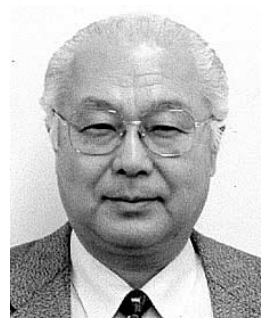

Toshitaka Idehara was born in Ibara, Japan, on April 15, 1940. He received the B.S. degree in mathematics from Kyoto University, Kyoto, Japan, in 1963 and the M.S. and D.S. degrees in physics from Kyoto University in 1965 and 1968 , respectively.

After graduating from Kyoto University, he joined Fukui University, Fukui, Japan, and was Lecturer from 1968 to 1970 and an Associate Professor from 1970 to 1990 . During this term, he worked in fundamental plasma physics. After 1979, his interest was directed toward the development of high-frequency gyrotrons. From 1990 to 1999, he was a Professor in the Applied Physics Department, Fukui University, and from 1992 to 1999, he was Head of Laboratory for Application of Superconducting Magnet, Fukui University. Since 1999, he has been a Professor and the Director of the Research Center for Development of Far-Infrared Region, Fukui University. His current research interests include development of frequency tunable, submillimeter wave gyrotrons and their applications to plasma diagnostics and material physics.

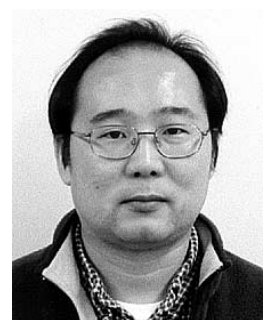

Seitaro Mitsudo was born in Okayama, Japan, on April 17, 1965. He received the B.S., M.S. and D.S. degrees in physics from Okayama University in 1989, 1991 and 1994, respectively.

After graduating from Okayama University, he joined the Institute for Materials Research of Tohoku University, Sendai, Japan. From 1994 to 1998, he was Research Associate. During this term, he worked in high field magnetism. From 1998 to 1999, he was with the Applied Physics Department of Fukui University, Fukui, Japan, as an Associate Professor. Since 1999, he has been an Associate Professor of the Research Center for Development of Far-Infrared Region, Fukui University. His current research interests include development of frequency tunable, submillimeter wave gyrotrons and their application for submillimeter wave ESR.

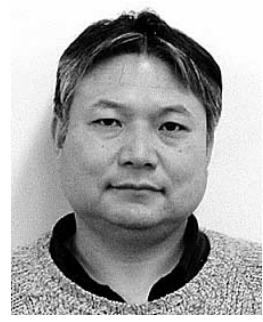

Isamu Ogawa was bone in Hamada, Japan, on August 15, 1955. He received the B.S. and M.S. degrees in physics from Ehime University, Matsuyama, Japan, in 1979 and 1981, respectively. He received the D.S. degree in physics from Nagoya University, Nagoya, Japan in 1987.

After graduating from Nagoya University, he joined Fukui University, Fukui, Japan. From 1989 to 1991, he was a Research Associate. Since 1991, he has been an Associate Professor in the Cryogenic Laboratory, Fukui University. His interest is directed to the system converting the output of a high-frequency gyrotron into a Gaussian beam and its application to the plasma scattering measurement. 